\title{
Surface disorder production during plasma immersion implantation and high energy ion implantation
}

\author{
M.A. El-Sherbiny ${ }^{\text {a,1 }}$, N.Q. Khánh ${ }^{a}$, H. Wormeester ${ }^{b}$, M. Fried ${ }^{a}$, T. Lohner ${ }^{\text {a }}{ }^{*}$, I. Pintér ${ }^{\text {a }}$, \\ J. Gyulai a \\ ${ }^{\text {a } K F K I ~ R e s e a r c h ~ I n s t i t u t e ~ f o r ~ M a t e r i a l s ~ S c i e n c e, ~ P . O . ~ B o x ~ 49, ~ H-I 525 ~ B u d a p e s t, ~ H u n g a r y ~}$ \\ ${ }^{\circ}$ Faculty of Applied Physics, University of Twente, P.O. Box 217, 7500 AE Enschede, The Netherlands
}

\begin{abstract}
High-depth-resolution Rutherford Backscattering Spectrometry (RBS) combined with channeling technique was used to analyze the surface layer formed during plasma immersion ion implantation (PIII) of single crystal silicon substrates. Single wavelength multiple angle of incidence ellipsometry (MAIE) was applied to estimate the thickness of the surface layer. The thickness of the disordered layer is much higher than the projected range of $P$ ions and it is comparable with that of protons.

Another example of surface damage investigation is the analysis of anomalous surface disorder created by $900 \mathrm{keV}$ and 1.4 MeV Xe implantation in $\langle 100\rangle$ silicon. For the $900 \mathrm{keV}$ implants the surface damage was also characterized with spectroellipsometry (SE). Evaluation of ellipsometric data yields thickness values for surface damage that are in reasonable agreement with those obtained by RBS.
\end{abstract}

\section{Introduction}

Plasma immersion implantation (PIII) is a promising method to fabricate very shallow junctions and this can be a significant contribution to the development of solar cell fabrication processes. In conventional photovoltaic devices the junction is formed by diffusion followed by a partial etch back step in order to achieve the required junction depth. For process optimization it is necessary to characterize the near surface region of the silicon after the PIII process. In this study, the phosphorous depth profile and the thickness of damaged surface layer were determined by Rutherford Backscattering Spectrometry (RBS) and the surface layer was probed using single wavelength multiple angle of incidence ellipsometry (MAIE).

The details of disorder formation in ion irradiated silicon have been the subject of extensive investigations motivated by fundamental and technological reasons. The properties of defects generated by ion irradiation determine the basic features of implantation-related processes such as ion beam induced layer-by-layer recrystallization and amorphization.

Ion irradiation induced anomalous surface disordering was observed in several cases [1-3]. In this case, anoma-

\footnotetext{
* Corresponding author. Tel. +3611699499 , ext. 2454 , fax +3611550694, e-mail nacs@rmhi.kfhi.hu.

${ }^{1}$ On leave from Faculty of Science, Al-Azhar University, Cairo, Egypt.
}

lous means that this disorder is far from that in the depth of the projected range. Earlier we saw this phenomenon in boron implanted silicon using a combination of single-wave ellipsometry and high-depth-resolution RBS $[4,5]$.

Ion bombardment induced surface damage can be observed in sapphire too. Considerably larger surface peaks from $\mathrm{Al}$ and $\mathrm{O}$ were found after implanting sapphire with $300 \mathrm{keV} \mathrm{Cr}$ ions than in the virgin crystal [6], and recently, an increased surface peak was detected due to $150 \mathrm{keV}$ Co ion bombardment in sapphire [7].

The applicability of spectroellipsometry (SE) for the nondestructive determination of ion-implantation induced damage profiles was demonstrated in detail by several research groups $[1,2,8-11]$. It was found that the thickness of the surface-damaged silicon layer (beneath the native oxide layer) created by nitrogen ion bombardment increased monotonicly with increasing implantation dose [12]. The amorphization rate at the surface was found to be proportional to the nuclear stopping power for $\mathrm{N}, \mathrm{Si}$ and Ar implantations [13].

In the present paper we report on an RBS and SE study of $\mathrm{Xe}$ implantation induced anomalous surface amorphization.

\section{Experimental}

For PIII experiments, a single wafer, parallel plate Reactive Ion Etching chamber was used, with an excitation 
frequency of $13.56 \mathrm{MHz}$ and a maximum power density of $3 \mathrm{~W} / \mathrm{cm}^{2}$. For PIII a gas mixture of $0.5 \% \mathrm{PH}_{3}$ in $\mathrm{Ar}$ at a flow rate of $33 \mathrm{sccm}$ and a pressure of $0.8 \mathrm{mbar}$ was applied. The bias voltage was $900 \mathrm{~V}$.

To investigate the surface amorphization process, 900

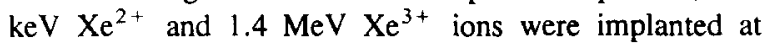
room temperature into $\langle 100\rangle$ silicon. The implantation was performed into as delivered Wacker single-crystal silicon at the Central Research Institute for Physics, Budapest. The implantation was done at room temperature with a current density of about $20-25 \mathrm{nA} / \mathrm{cm}^{2}$.

RBS and channeling techniques with 1.5 or $2.95 \mathrm{MeV}$ $\mathrm{He}^{+}$ions were used in the experiments. Two detectors were placed to detect ions scattered through $165^{\circ}$ and $97^{\circ}$ (i.e. with a glancing exit angle of $7^{\circ}$ to the surface). In the latter geometry, the depth resolution was better than $5 \mathrm{~nm}$ [14]. To evaluate the spectra we used the RBX code written by Kótai [15], which can also handle channeled spectra.

In reflection ellipsometry the experimental result is given by:

$\rho=R_{\mathrm{p}} / R_{\mathrm{s}}=\tan \Psi \exp (\mathrm{i} \Delta)$,

where $\rho$ is the complex reflectance ratio. $R_{\mathrm{p}}$ and $R_{\mathrm{s}}$ are the complex amplitude reflection coefficients for the parallel (p) and perpendicular (senkrecht, s) to the plane of incidence polarizations respectively, $\tan \Psi$ is the intensity ratio and $\Delta$ is the relative phase difference. With a rotating element ellipsometer one can determine directly $\tan \psi$ and $\cos \Delta$. Using manual ellipsometry one can measure $\psi$ and $\Delta$. The SE spectra were obtained at Twente University in the range of $300-650 \mathrm{~nm}$ with a rotating polarizer ellipsometer. A manual ellipsometer was used for MAIE to estimate the thickness and the refractive index of the surface layer of PIII samples. The wavelength was 632.8 $\mathrm{nm}$ ( $\mathrm{He}-\mathrm{Ne}$ laser).

\section{Results and discussion}

\subsection{Plasma immersion implantation}

Figs. la and lb present the high-depth resolution RBS spectra of $\alpha$ 's scattered through $97^{\circ}$ and $165^{\circ}$ respectively, for a PIII sample. The depth of phosphorus atoms is shallow enough to be analyzed by RBS. The surface region of the sample consists of an oxide layer and a disordered layer. The evaluation yields a thickness of $19 \mathrm{~nm}$ for the oxide layer (assumed to be $\mathrm{SiO}_{2}$ ) and a thickness of $15 \mathrm{~nm}$ for the disordered Si layer. The thickness of the disordered layer is much greater than the projected range of $\mathrm{P}^{+}$ions $(3.5 \mathrm{~nm})$ and is comparable with that of protons $(14 \mathrm{~nm})$. The amount of $P$ atoms was found to be $8.5 \times 10^{15}$ atoms $/ \mathrm{cm}^{2}$. However, the majority of $P$ atoms are located in the oxide layer.
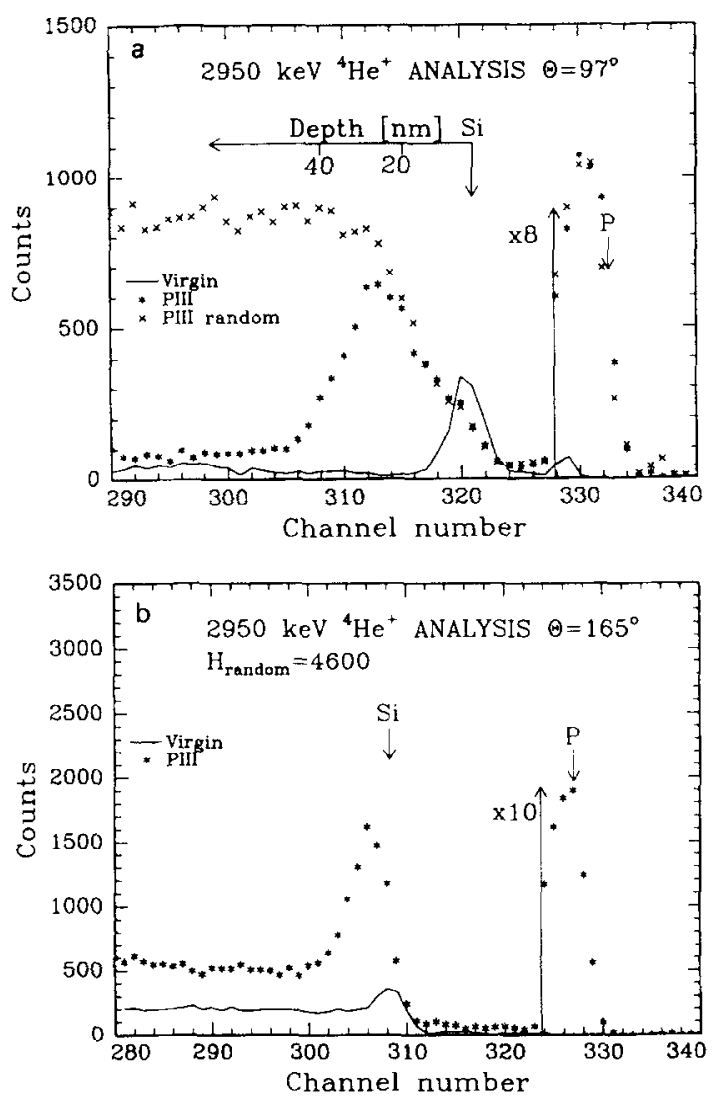

Fig. 1. (a) Random and $\langle 100\rangle$-aligned high-depth-resolution RBS spectra for the PIII sample recorded with the detector placed at $97^{\circ}$ scattering angle. (b) Random and $\langle 100\rangle$-aligned normal resolution spectra for the PIII sample recorded with the detector placed at $165^{\circ}$ scattering angle.

MAIE data along with the analysis results are shown in Fig. 2 for the PIII sample. The optical model consists of a surface layer and a crystalline silicon substrate. The thickness value of $35 \mathrm{~nm}$ is comparable with that deduced from RBS measurements. The low value obtained for the refractive index of the surface layer indicates the presence of oxide and a possible surface roughness.

\subsection{Anomalous surface amorphization}

Figs. $3 a$ and $3 b$ present the high-depth resolution RBS spectra recorded with a detector placed at a scattering angle of $97^{\circ}$ and also a spectra recorded with a detector placed at a scattering angle of $165^{\circ}$, respectively. They include spectra for the series of $900 \mathrm{keV}$ xenon implanted samples and for the virgin sample. In accordance with projected range calculations, significant buried disorder is observable around a depth of $280 \mathrm{~nm}$ (Fig. 3b). The energetic xenon ions are shown to have created a disorder at the silicon surface. The thicknesses of the surface oxide and disordered layers in units of atoms $/ \mathrm{cm}^{2}$ were deduced 


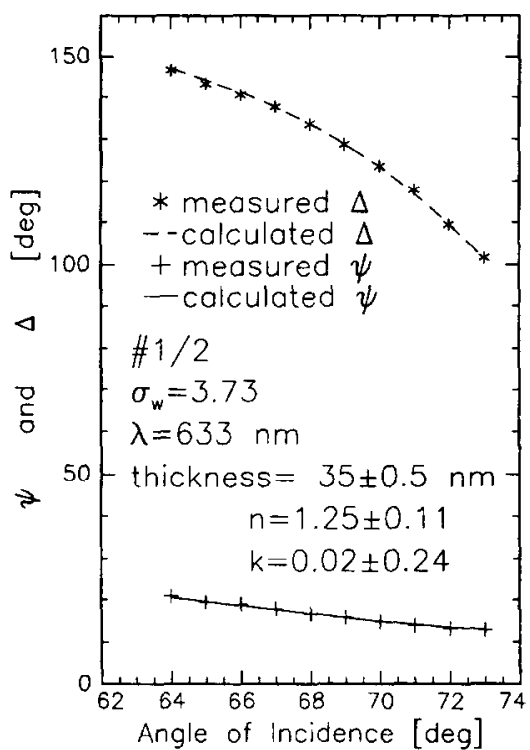

Fig. 2. Measured single wavelength MAIE data for the PIII sample together with the result of evaluation; $n$ is the refractive index, $k$ is the extinction coefficient, $\sigma_{\mathrm{w}}$ characterizes the quality of the fit.

from the oxygen peak and the surface damage peak of the spectra in Fig. 3a, respectively. The thicknesses in unit of $\mathrm{nm}$ were calculated assuming the surface disorder was totally amorphous.

For the evaluation of SE data, the $420 \mathrm{~nm}$ wavelength was chosen as an upper limit because in this case the optical penetration depth (even in crystalline $\mathrm{Si}$ ) is not larger than $200 \mathrm{~nm}$. The optical model consists of a native oxide layer, a thin amorphous silicon layer and a slightly damaged semiinfinite layer. For the analysis of SE data we used the conventional method of assuming an appropriate optical model and fitting the model parameters (layer thicknesses and volume fraction of the constituents in the slightly damaged semiinfinite layer) by linear regression. It is important to note that we used the complex dielectric function of implanted amorphous silicon [16]. The slightly damaged semiinfinite layer was modelled as a mixture of
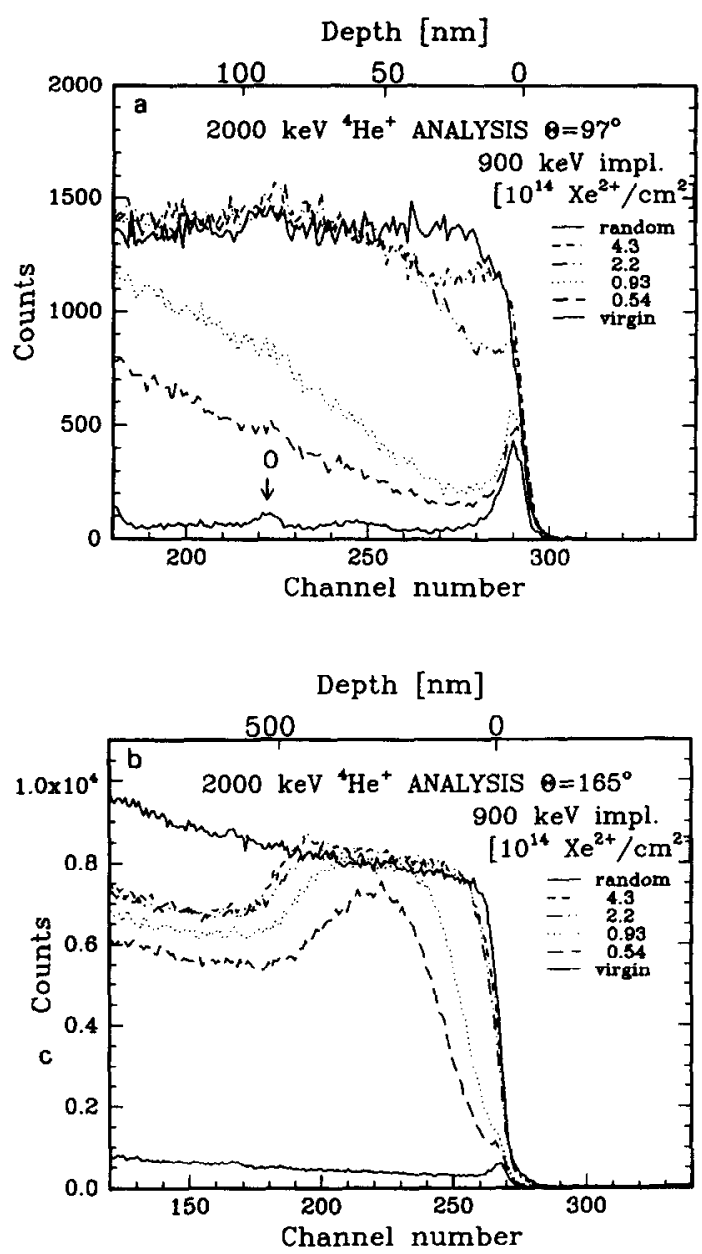

Fig. 3. (a) Random and $\langle 100\rangle$-aligned high-depth-resolution RBS spectra for $900 \mathrm{keV}$ xenon implanted samples recorded with the detector placed at $97^{\circ}$ scattering angle. (b) Random and $\langle 100\rangle$ aligned normal resolution spectra for $900 \mathrm{keV}$ xenon implanted samples recorded with the detector placed at $165^{\circ}$ scattering angle.

crystalline silicon and fine-grain polycrystalline silicon [17], i.e. the complex refractive index of the slightly disordered layer was calculated by Bruggeman effective

Table 1

Implantation conditions together with the results of RBS/channeling analysis and SE fitting procedure. $D_{\mathrm{a}-\mathrm{Si}}$ is the thickness of the surface amorphous silicon, $D_{\text {ox ide }}$ is the thickness of native oxide, $\sigma$ is the unbiased estimator for $\mathrm{SE}$

\begin{tabular}{|c|c|c|c|c|c|c|}
\hline \multirow{2}{*}{$\begin{array}{l}\text { Ion } \\
\text { (Energy) }\end{array}$} & \multirow{2}{*}{$\begin{array}{l}\text { Dose } \\
10^{13} / \mathrm{cm}^{2}\end{array}$} & \multicolumn{2}{|l|}{$D_{\mathrm{a}-\mathrm{Si}}[\mathrm{nm}]$} & \multicolumn{2}{|l|}{$D_{\text {oxide }}[\mathrm{nm}]$} & \multirow[t]{2}{*}{$\sigma\left(10^{-3}\right)$} \\
\hline & & $\overline{S E}$ & RBS & $\overline{\mathrm{SE}}$ & RBS & \\
\hline & Virgin & - & - & $2.2 \pm 0.05$ & $1.4 \pm 0.2$ & 4.2 \\
\hline $\begin{array}{l}\mathrm{Xe} \\
(900 \mathrm{keV})\end{array}$ & $\begin{array}{l}5.4 \\
9.3\end{array}$ & $\begin{array}{l}0.6 \pm 0.05 \\
1.1 \pm 0.05\end{array}$ & $\begin{array}{l}1.09 \pm 0.25 \\
1.46 \pm 0.36\end{array}$ & $\begin{array}{l}2.3 \pm 0.05 \\
2.0 \pm 0.05\end{array}$ & $\begin{array}{l}1.0 \pm 0.3 \\
1.7 \pm 0.5\end{array}$ & $\begin{array}{l}2.6 \\
2.3\end{array}$ \\
\hline $\begin{array}{l}\mathrm{Xe} \\
(1.4 \mathrm{MeV})\end{array}$ & $\begin{array}{l}4.2 \\
5.0 \\
6.7 \\
7.5\end{array}$ & $\begin{array}{l}- \\
- \\
- \\
-\end{array}$ & $\begin{array}{l}0.4 \pm 0.1 \\
0.7 \pm 0.1 \\
1.1 \pm 0.1 \\
1.0 \pm 0.1\end{array}$ & $\begin{array}{l}- \\
- \\
- \\
-\end{array}$ & $\begin{array}{l}1.3 \pm 0.2 \\
1.5 \pm 0.2 \\
1.5 \pm 0.2 \\
1.4 \pm 0.2\end{array}$ & $\begin{array}{l}- \\
- \\
- \\
-\end{array}$ \\
\hline
\end{tabular}


medium approximation using crystalline and fine-grain polycrystalline silicon as end-points.

The data for the implanted samples was analyzed using a FORTRAN program developed at the Pennsylvania State University. We used grid search before fitting. The goodness of the fit is estimated by the unbiased estimator:

$$
\begin{aligned}
\sigma= & \frac{1}{2 N-p-1} \\
& \times \sqrt{\sum_{j=1}^{N}\left[\left(\cos \Delta_{j}^{\text {exp }}-\cos \Delta_{j}^{\text {calc }}\right)^{2}+\left(\tan \Psi_{j}^{\exp }-\tan \Psi_{j}^{\text {calc }}\right)^{2}\right]} .
\end{aligned}
$$

where $N$ is the number of wavelengths and $p$ is the number of fitted parameters.

To study anomalous surface disorder (assuming a thin amorphous layer) SE is extremely useful because of its sensitivity.

Measured ellipsometric spectra of silicon samples implanted with xenon ions together with the results of multiparameter fitting are shown in Fig. 4. For comparison, a reference spectrum of virgin (unimplanted) silicon was also presented.

Table 1 summarizes the layer thickness values resulting from the evaluation of RBS and SE measurements. For
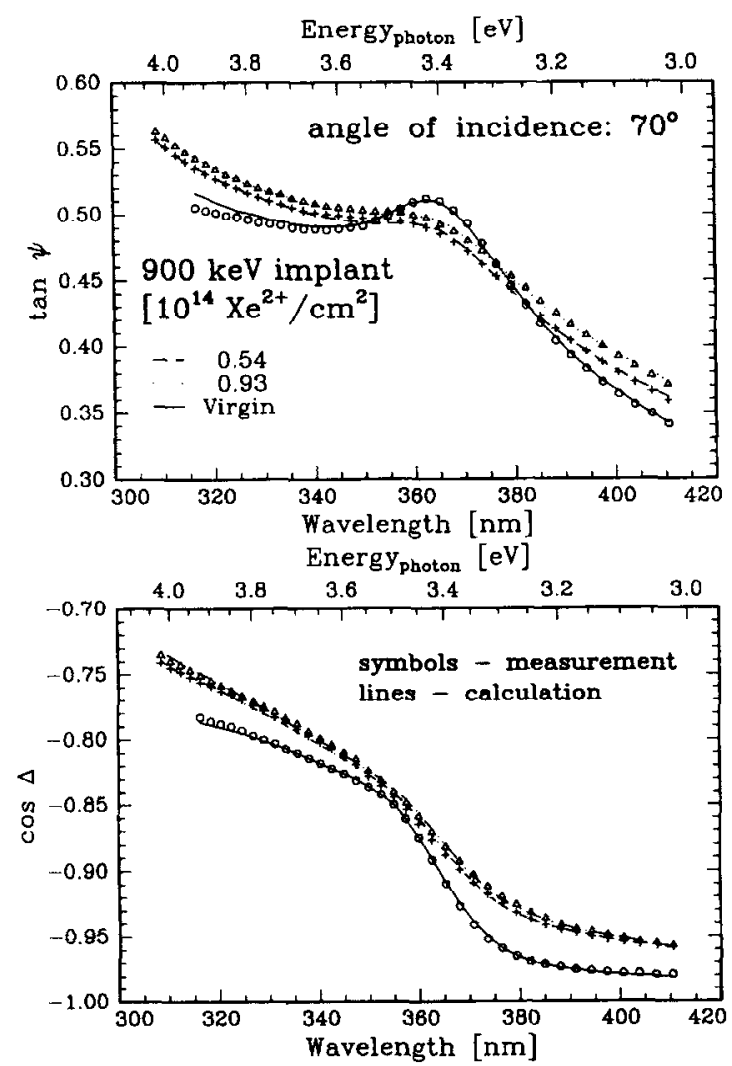

Fig. 4. Results of SE fitting for the $900 \mathrm{keV}$ xenon implanted silicon samples. The angle of incidence was $70^{\circ}$.
Table 2

The dependence of the surface amorphization rate (normalized amorphous layer thickness) on the nuclear energy deposition. The values for N, Ar and Si ions were taken from Ref. [13]

\begin{tabular}{lrll}
\hline Ion & $\begin{array}{l}\text { Energy } \\
{[\mathrm{keV}]}\end{array}$ & $\begin{array}{l}\text { Nuclear stopping } \\
\text { power } \\
{[\mathrm{eV} / \AA]}\end{array}$ & $\begin{array}{l}\text { Surface amorphization rate } \\
\text { (normalized amorphous layer } \\
\text { thickness) } \\
{\left[\mathrm{nm} / 10^{14} \text { atom } / \mathrm{cm}^{2}\right]}\end{array}$ \\
\hline $\mathrm{N}$ & 200 & 4.1 & $0.035 \pm 0.0028$ \\
$\mathrm{Ar}$ & 800 & 18.1 & $0.19 \pm 0.0086$ \\
$\mathrm{Si}$ & 200 & 20.5 & $0.22 \pm 0.018$ \\
$\mathrm{Xe}$ & 1400 & 145 & $1.39 \pm 0.12$ \\
$\mathrm{Xe}$ & 900 & 169 & $1.68 \pm 0.19$ \\
\hline
\end{tabular}

RBS analysis we used the density of silicon $\left(5 \times 10^{22}\right.$ atoms $/ \mathrm{cm}^{3}$ ) to calculate the thickness of the surface disordered layer. Evaluation of ellipsometric data yields thickness values for surface damage that are in reasonable agreement with those obtained by RBS. However, some differences can be observed in thickness values. This reveals that SE is sensitive to surface damage induced by heavy ion implantation, but for precise evaluation a more adequate optical model is needed.

The difference in the thickness of the surface (native) oxide can be explained on the basis of morphological and compositional properties of this layer. We use the refractive index of the $\mathrm{SiO}_{2}$ but this surface is possibly a roughened and unstoichiometric one. SE determines an effective thickness but RBS gives atoms $/ \mathrm{cm}^{2}$.

To relate the surface amorphization rate to the amount of nuclear cnergy deposited, the increase in the amorphized surface layer thickness with respect to ion dose was calculated by fitting a straight line to the points belonging to a given implantation energy. The dependence of the surface amorphization rate (normalized amorphous layer thickness) on the nuclear energy deposited at the surface is shown in Table 2. The nuclear stopping power at the implantation energy was calculated using TRIM code [18]. For comparison, the values for $\mathrm{N}, \mathrm{Ar}$ and $\mathrm{Si}$ ions were taken from Ref. [13]. The surface amorphization rate was found to be proportional to the nuclear stopping power for $\mathrm{N}, \mathrm{Si}$ and Ar implantations [13].

\section{Conclusion}

It was demonstrated that RBS combined with channeling has the potential to yield the concentration of phosphorous atoms, the thickness of the surface oxide, and the thickness of the disordered layer in silicon samples which were processed by PIII. We determined the surface amorphization rate for the case of high energy Xe ion implantation into Si. We think the surface amorphization is an analogous process to Ion Beam Induced Epitaxial Crystallization and Ion Beam Induced Amorphization [19-23]. 
The most probable explanation for the surface disordering is the diffusion of point defects to the surface.

Spectroscopic ellipsometry, high-depth-resolution Rutherford backscattering and channeling have been used to examine the surface damage formed by room temperature Xe implantation into silicon. A multiparameter fitting procedure of ellipsometric data was applied to evaluate the surface damage. The results demonstrate the applicability of spectroscopic ellipsometry together with a proper optical model construction for probing surface damage.

\section{Acknowledgements}

M.A. El-Sherbiny is grateful to the Ministry of Higher Education of Egypt for a scholarship during his research work. Partial support from OTKA grants (No. 3265, No. F4378 and No. T016821) is greatly appreciated. This work was partially supported by JOULE-CT92-0179 MULTICHESS2 linked with PECO-PL932049 project. For his assistance with implantation Ing. J. Waizinger is acknowledged. The authors wish to thank the team operating the accelerator for their help in experimental procedures.

\section{References}

[1] M. Erman, J.B. Theeten, Surf. Interface Anal. 4 (1982) 98.

[2] M. Erman, J.B. Theeten, Surf. Sci. 135 (1983) 353.

[3] G.Q. Yang. N.Q. Khanh, M. Fried, E. Kótai, V. Schiller, L.C. Lu, J. Gyulai, S.C. Zou, Radiat. Eff. Def. Sol. 115 (1990) 183

[4] T. Lohner, G. Mezey, E. Kótai, F. Pászti, A. Manuaba, J. Gyulai, Nucl. Instr. and Meth. 209/210 (1983) 615.

[5] T. Lohner, E. Kótai, F. Pászti, A. Manuaba, M. Fried, J. Gyulai, J. Radioanal. and Nucl. Chemistry 83/1 (1984) 75.
[6] H. Naramoto, C.W. White, J.M. Williams, C.J. McHargue, O.W. Holland, M.M. Abraham and B.R. Appleton, J. Appl. Phys. 54 (1983) 683.

[7] E. Németh-Jároli, E. Szilágyi, N.Q. Khánh and P. Pécz, these Proceedings (IBA-12), Nucl. Instr. and Meth. B 118 (1996) 123.

[8] P.J. McMarr, K. Vedam and J. Narayan, J. Appl. Phys. 59 (1986) 694

[9] J. Vanhellemont, P. Roussel and H.E. Maes, Nucl. Instr. and Meth. B 55 (1991) 183.

[10] M. Fried, T. Lohner, W.A.M. Aamink, L.J. Hanekamp and A. van Silfhout, J. Appl. Phys. 71 (1992) 2835.

[11] S. Lynch, M. Murtagh, G.M. Crean, P.V. Kelly, M. O'Connor and C. Jeynes, Thin Solid Films 233 (I993) 199.

[12] T. Lohner, E. Kótai, N.Q. Khánh, Z. Tóth, M. Fried, K. Vedam, N.V. Nguyen, L.J. Hanekamp and A. van Silfhout, Nucl. Instr. and Meth. B 85 (1994) 335.

[13] T. Lohner, M.A. El-Sherbiny, N.Q. Khánh, M. Fried, H. Wormeester and J. Gyulai, Proc. 9th Int. Conf. on Ion Beam Modification of Materials (IBMM'95) Australia, February 1995, eds. J.S. Williams, R.G. Elliman, M.C. Ridgway (Elsevier, Amsterdam, 1996) p. 797.

[14] G. Mezey, E. Kótai, T. Lohner, T. Nagy, J. Gyulai and A. Manuaba, Nucl. Instr. and Meth. 149 (1978) 235.

[15] E. Kótai, Nucl, Instr. and Meth. B 85 (1994) 588.

[16] M. Fried, T. Lohner, W.A.M. Aarnink, L.J. Hanekamp and A. van Silfhout, J. Appl. Phys. 71 (1992) 5260.

[17] G.E. Jellison Jr., M.F. Chisholm and S.M. Gorbatkin, Appl. Phys. Lett. 62 (1993) 3348.

[18] J.F. Ziegler, J.P. Biersack, U. Littmark, The Stopping and Range of Ions in Solids (Pergamon, New York, 1985).

[19] F. Priolo and E. Rimini, Mater. Sci. Rep. 5 (1990) 319.

[20] J.S. Williams, R.G. Elliman, W.L. Brown and T.E. Seidel, Phys. Rev. Lett. 55 (1985) 1482.

[21] J. Linross; G. Holmen and B. Svensson, Phys. Rev. B 32 (1985) 2770.

[22] A. Battaglia, F. Priolo and E. Rimini, Appl. Surf. Sci. 56-58 (1992) 577.

[23] J. Nakata, Phys. Rev. B 43 (1991) 14643. 